\title{
Plasmid-mediated Colistin Resistance: What Do We Know?
}

\author{
Tanise V. Dalmolin ${ }^{1,2}$, Daiana de Lima-Morales', Afonso L. Barth ${ }^{1,2 *}$ \\ 'LABRESIS - Laboratório de Pesquisa em Resistência Bacteriana, Hospital de Clínicas de Porto Alegre, Porto Alegre, RS, Brazil \\ ${ }^{2}$ Programa De Pós-Graduação em Ciências Farmacêuticas, Faculdade de Farmácia, Universidade Federal do Rio Grande do Sul, Porto Alegre, RS, Brazil
}

Article Info

\section{Article Notes}

Received: June 13, 2018

Accepted: August 21, 2018

\section{${ }^{*}$ Correspondence:}

Dr. Afonso L. Barth, Laboratório de Pesquisa em Resistência Bacteriana - LABRESIS, Hospital de Clínicas de Porto Alegre, Ramiro Barcelos 2350, Porto Alegre-RS, Brazil; Tel.: +55 5133598607; Fax: +55 5133598760; Email: albarth@ hcpa.edu.br

${ }^{0} 2018$ Barth AL. This article is distributed under the terms of the Creative Commons Attribution 4.0 International License.

\section{Keywords}

mcr-1 gene

Polymyxins

Antimicrobial resistance

Enterobacterales

Gram-negative bacteria

Colistin
Abstract

Polymyxins (polymyxin E/colistin and polymyxin B) are considered the lastresort therapy against carbapenem-resistant Enterobacterales; however, the resistance of Enterobacterales to polymyxins is increasing worldwide. Until 2015, this resistance was related to chromosomal mutations, but in November 2015, it was described in China the transferable colistin resistance in animals and humans isolates of $E$. coli and $K$. pneumoniae, mediated by the $m c r-1$ gene (mobile colistin resistance), located in a plasmid. Following the first description of the mcr-1 gene, it has been reported in several regions of the world, in different bacterial species, from different sources and others $\mathrm{mcr}$ variants have been described. Moreover, the co-occurence of the $\mathrm{mcr}$ gene and other antimicrobial resistance genes was reported. This discovery changed the scenario of resistance to polymyxins, as this gene could be promptly disseminated among Gram negative bacilli becoming a major concern for public health. This review summarizes recent data about the plasmid-borne mor colistin resistance gene.

\section{Introduction}

Antimicrobial resistance is considered as a major public health threat to human health mainly due to the widespread of carbapenemresistant Enterobacterales (CRE) in the last decade. The colistin (polymyxin E) and polymyxin B belong to the class of polymyxins, which are considered the last-resort therapy against CRE; however, the resistance of Enterobacterales to polymyxins is increasing worldwide ${ }^{1,2,3}$. Overall, colistin resistance in Enterobacterales (excluding species with intrinsic resistance) is around $0.67-1.6 \%$, with low rates in Escherichia coli (0.2-0.6\%), moderate rates in Klebsiella pneumoniae (1.5-6.8\%), and much higher rates in Enterobacter spp. (13.9-20.1\%). Considering other resistance mechanisms associated with colistin resistance such as ESBLs the percentage of co-producers is: $2.3-5.5 \%$ for Enterobacterales $(>11.5 \%$ are K. pneumoniae); for carbapenem-non-susceptible isolates the number is higher: $4.5-16.3 \%$ for Enterobacterales (32\% are K. pneumoniae) and even higher is the number found for carbapenemase-producers, $6.2-12.0 \%$ for Enterobacterales (14$36.6 \%$ are K. pneumoniae) ${ }^{4}$.

As mentioned above, polymyxins are active against most Enterobacterales and also have significant activity against nonfermentative Gram-negative bacteria such as Pseudomonas aeruginosa and Acinetobacter spp. Some Gram-negative species are naturally resistant to polymyxins: Proteus spp., Morganella morganii, Providencia spp., Serratia marcescens, Burkholderia mallei, Burkholderia cepacia complex, Chromobacterium spp., 
Edwardsiella spp., Brucella, Legionella, Campylobacter and Vibrio cholerae. ${ }^{5}$ Additionally, the polymyxins are not active against Gram-negative cocci, Gram-positive and anaerobic bacteria ${ }^{5,6}$. The natural resistance mechanism is hypothesized to be promoted by constitutive gene expression which adds cationic molecules to the LPS, leading to decreased affinity at the polymyxin site of action on the LPS, differently in Gram-positive bacteria lack of an LPS containing outer cell membrane promotes the resistance ${ }^{7}$.

The colistin was obtained in 1947 from a soil bacteria Paenibacillus polymyxa subsp. colistinus ${ }^{8}$ and has been cleared to use since 1959 by the Food and Drug Administration (FDA) for the treatment of infectious diseases caused by Gram-negative bacteria. However, in clinical use, colistin proved to be nephrotoxic and their use has declined from the early 1970 s to the early 2000 s, being replaced by more active and less-toxic antibiotics such as the aminoglycosides, quinolones and beta-lactams. However, due to increasing prevalence of multiresistant Gram-negative bacteria, the colistin were re-introduced into clinical practice as a valuable therapeutic option ${ }^{5,9}$.

Unlike in human medicine, in veterinary medicine, colistin has been used extensively, and without interruption, for decades for the treatment and prevention of infectious diseases by Enterobacterales, as well as growth promoter in poultry and pigs ${ }^{2,5,9}$. However, due to the description of the colistin resistance mediated by a mobile genetic element and its widespread in animals in China in 2015, the Ministry of Agriculture of China decided to ban colistin as a feed additive for animals in 2016. Other countries also decided to ban colistin as growth animal promoter, due to the imminent impact on polymyxin resistance in human health ${ }^{10}$.

The mechanism of action of polymyxins (colistin and polymyxin B) occurs by the electrostatic binding of the molecule to the lipopolysaccharide (LPS) and phospholipids in the outer membrane of Gram-negative bacteria. The polymyxins destabilize the LPS through the exchange of ions $\left(\mathrm{Ca}^{+2}\right.$ and $\mathrm{Mg}^{+2}$ ), increasing the permeability of the bacterial membrane, promoting the extravasation of the cytoplasmic content and consequent bacterial death. Despite the fact that the initial target for polymyxin is the LPS, the exact mode of action of polymyxins is still not totally clear. There are other possible mechanisms of action described for polymyxins: the endotoxin effect (during cell lysis the endotoxin molecule - the lipid A which is the main component of the LPS) is released and the polymyxins are able to bind and neutralize it and the inhibition of respiratory enzymes (type II NADH-quinone oxidoreductases [NDH-2]) in the bacterial membrane ${ }^{5,11}$.

Until 2015, the reports of polymyxin resistance were all due to chromosomal mutations. These chromosomal mutations lead to modification of the LPS via cationic substitution that alter the lipid A, decreasing its affinity for the polymyxins, similarly to that observed in bacteria with intrinsic resistance to polymyxins. There are several mutation in genes and operons which are involved in the modification of the LPS: (i) mutations in genes ( $p m r C, p m r E$ and pmrHFIJKLM) responsible for synthesis of cationic groups and their addition to the LPS; (ii) mutations in regulatory genes that encode proteins involved in the PmrAB system in K. pneumoniae, Enterobacter aerogenes and Salmonella enterica ( $p m r A$ and $p m r B$ genes) and the PhoPQ system in K. pneumoniae and E. coli (phoP and phoQ genes); (iii) and mutations in the regulators of these systems as the $\operatorname{mgr} B$ gene in Klebsiella spp. (regulates the PhoPQ system) and $\operatorname{cr} A B$ operon in $K$. pneumoniae (regulates the PmrAB system) ${ }^{12}$.

In November 2015, Liu and colleagues described the transferable colistin resistance in animals and humans isolates of E. coli and K. pneumoniae recovered in China, mediated by the $m c r-1$ gene (mobile colistin resistance). The $m c r-1$ gene is located in a plasmid and its discovery changed the scenario of resistance to polymyxins, as this gene could be promptly disseminated among Gramnegative bacilli becoming a major concern for public health. MCR-1 protein is a member of the phosphoethanolamine transferase family, its acquisition promotes the addition of phosphoethanolamine (PEtN) to lipid A, similarly to the chromosomal mutations, resulting in reduction of polymyxin affinity ${ }^{1}$.

\section{Epidemiology of Plasmid-mediated Colistin Resistance}

Following the first description of the $m c r-1$ gene, it has been reported in several regions of the world, including countries of Asia, Africa, Europe and America ${ }^{2,12,13}$. Further studies indicated that bacteria carrying the $m c r-1$ gene were identified in the 1980's in China. The earliest report of the $m c r-1$ gene among isolates from humans is from 2008 and it was described in a Shigella sonnei from Vietnam. These findings indicate that mcr-1 gene has existed in Enterobacterales but remained not identified for a long time $e^{9,14}$.

The $m c r-1$ gene has been reported in several species of Enterobacterales but mostly in E. coli. The occurrence in Salmonella, Klebsiella, Shigella, Vibrio and Enterobacter was only sporadically reported, but indicates interspecies gene transfer ${ }^{2,12}$.

The mcr-1 gene has been mainly detected in $E$. coli isolates obtained from animals (livestock, wild animals and food of animal origin). Farm animals, in particular pigs and chickens, have been considered as reservoirs for $E$. coli isolates carrying the $m c r-1$ gene. In humans, Enterobacterales carrying the mcr-1 gene have 
been obtained from clinical samples, as well as from asymptomatic patients, including international travellers. The possible dissemination of the $\mathrm{mcr}$ - 1 gene from bacteria obtained from animals to bacteria from humans is a serious concern, mainly whether this gene could be transferred into carbapenemase producing Enterobacterales ${ }^{2,9,12}$.

MCR-1 producers may exhibit low level resistance to colistin or polymyxin B (minimum inhibitory concentration [MIC] of $4 \mu \mathrm{g} / \mathrm{mL}$ ) and a few cases of isolates carrying the $m c r-1$ gene which are susceptible to colistin have already been described ${ }^{15,16}$. This may contribute to the silent dissemination of $m c r-1$ gene harbouring isolates. Other aspects may also play an important role in the dissemination of the mcr-1 gene: the technical problems related to the detection of resistance to polymyxins and the fact that most laboratories only evaluate the susceptibility profile to polymyxins among carbapenem non-susceptible isolates ${ }^{15}$.

One explanation for the reported $m c r-1$ positive but colistin susceptible isolates was that the gene was truncated as described in a Shigella sonnei isolate ${ }^{17}$. Interestingly, the truncated mcr-1 gene could be re-activated after conjugation experiments resulting in a colistin resistant phenotype $^{18}$. Conversely, the report of an E. coli colistin susceptible and $m c r-1$ positive with an intact gene indicates that the gene truncation is not the only mechanism related to the susceptibility to polymyxins among $m c r-1$ harboring isolates ${ }^{19}$.

According to an search assessment of the data at the GenBank (https://www.ncbi.nlm.nih.gov/genbank/ - $17^{\text {th }}$ of June 2018), $13 \mathrm{mcr}$-1 subgroups were already described in several countries, differing from $m c r-1$ by only one nucleotide: $m c r-1.2$ (K. pneumoniae from Italy $)^{20}, m c r-1.3$ (E. coli from China) ${ }^{21}$, mcr-1.4 (E. coli from China), mcr-1.5 (E. coli from Argentina), mcr-1.6 (Salmonella typhimurium from China) ${ }^{22}$, mcr-1.7 (E. coli from China), mcr-1.8 (E. coli from Brunei) ${ }^{23}$, mcr-1.9 (E. coli from China $)^{24}$, $m c r-$ 1.10 (Moraxella spp. from Great Britain) ${ }^{25}, \mathrm{mcr}^{2}-111$
(KY853650.1), mcr-1.12 (LC337668.1) and mcr-1.13 (E. coli from Italy $)^{26}$.

To date, eight $m c r$ variants have been described: $m c r-1$, $m c r-2, m c r-3, m c r-4, m c r-5, m c r-6, m c r-7$ and $m c r-8$ (Table 1). The $m c r-2$ gene is $1,617 \mathrm{bp}$ long, nine bases shorter than mcr-1 $(1,626 \mathrm{bp})$ and it has $76.7 \%$ nucleotide identity to $\mathrm{mcr}$ $1^{27}$. The $\mathrm{mcr}$ - 3 gene presented $45.0 \%$ and $47.0 \%$ nucleotide sequence identity to $m c r-1$ and $m c r-2$, respectively. MCR3 also showed $94.1 \%$ to $94.8 \%$ amino acid identity with proteins found in three Aeromonas species. Moreover, a truncated transposon element (TnAs2), which was characterized only in Aeromonas salmonicida, was located upstream of $m c r-3$. These findings suggest that $m c r-3$ gene in Enterobacterales might have originated from Aeromonas species $^{28}$. Unusually, the $m c r-4$ is inserted in a small and not self-conjugative plasmid. However, the addition of an auxiliary plasmid can promote conjugation ${ }^{29}$.

\section{Co-occurrence of $\mathbf{m c r}$ and Other Resistance Genes}

There have been reports of co-production of the mcr1 gene and other resistance genes in several bacterial species. The presence of the $m c r-1$ gene has been associated with ESBLs (CTX-M, SHV and TEM type) and AmpC cephalosporinase (CMY type). Moreover, there are descriptions of co-occurrence of the $m c r-1$ gene and quinolone resistance genes ( $q h r S$ and $\operatorname{aac}(6=)-I b-c r)^{9,15,33,34}$.

Isolates carrying $m c r-1$ and carbapenemase resistance genes have been described in Enterobacterales. Specifically, the co-production of $m c r-1$ and carbapenem resistance genes is of great concern, since polymyxins represent the "last-line" therapeutic option for an infection caused by a carbapenemase-producing isolate. In the literature the $m c r-1$ gene has been found mainly in isolates that produce the carbapenemase NDM in particular among E. coli isolates from animal and human origin ${ }^{35,36,37}$. However, it was also detected in Cronobacter sakazakii ${ }^{38}$ from animal origin and K. pneumoniae ${ }^{39}$ from human origin. Only one report about co-producer OXA-48 carbapenemase and $m c r-1^{40}$ and

Table 1. mor variants available at the GenBank database (update in July 17, 2018- https://www.ncbi.nlm.nih.gov/genbank/)

\begin{tabular}{|c|c|c|c|c|c|}
\hline Gene & Country & Origin & Species & Reference & Variants \\
\hline$m c r-1$ & China & $\begin{array}{l}\text { Retail meat from chicken and pig, and } \\
\text { inpatients }{ }^{a}\end{array}$ & $\begin{array}{l}\text { E. coli and } \\
\text { K. pneumoniae }\end{array}$ & 1 & $m c r-1.2-m c r-1.13$ \\
\hline$m c r-2$ & Belgium & Pig and bovine & E. coli & 27 & $m c r-2.2$ \\
\hline$m c r-3$ & China & Pig & E. coli & 28 & mcr-3.2-mcr-3.12 \\
\hline$m c r-4$ & Italy, Spain and Belgium & Pig & Salmonella enterica and $E$. coli & 29 & mcr-4.2 \\
\hline mcr-5 & Germany & $\begin{array}{l}\text { Faecal content of pigs and faecal } \\
\text { samples from pigs }\end{array}$ & $\begin{array}{l}\text { S. enterica subsp. enterica } \\
\text { serovar Paratyphi B }\end{array}$ & 30 & $m c r-5.2$ \\
\hline$m c r-6^{b}$ & Great Britain & Faecal contents of healthy pigs & Moraxella sp. & 25 & - \\
\hline mcr-7 & China & Chickens & K. pneumoniae & 31 & - \\
\hline$m c r-8$ & China & Pig, chicken and inpatient & K. pneumoniae & 32 & \\
\hline
\end{tabular}

aThe gene $m c r-1$ was isolated from several other origin, after its first publication from Liu et al., $2016^{1}$.

${ }^{\mathrm{b}} \mathrm{mcr}-2.2$ (1617 bp) has been renamed as $\mathrm{mcr}-6$. 
VIM carbapenemase and $m c r-1^{41}$ have been related in the literature, both from human sources. There are a few KPC and $m c r-1$ co-producers reported, in particular those genes were identified in $E$. coli $i^{42,43,44,45,46}$ and less frequently in $K$. pneumoniae isolates ${ }^{20,47,48}$.

\section{Genetic Context of the mcr Gene}

The first plasmid carrying the $m c r-1$ gene belonged to the plasmid incompatibility type Incl $2^{1}$. Others studies indicated that $m c r-1$ gene is not restricted to the IncI2 plasmid group and various plasmids may carry the $m c r-1$ gene including those belonging to the IncX4, InHI2, IncF, IncHI1, IncY and IncP ${ }^{33,49}$. These incompatibility groups of plasmids have been implicated in the global spread of others resistance genes among Enterobacterales from human and animal sources ${ }^{50,51}$. As the mcr-1 gene is not associated with a specific incompatibility group of plasmids, it can be considered that this molecular flexibility promotes global dissemination of the gene ${ }^{52}$.

Noteworthy, there are intriguing descriptions of coexistence of two $\mathrm{mcr}$ bearing plasmids in a single bacterial isolate. Nevertheless, the MIC for colistin of the isolates presenting more than one $\mathrm{mcr}$ gene in different plasmids was not increased ${ }^{52,53}$.

Often the mor gene is flanked by an insertion sequence designated ISAPI1 and the nikB gene, in this order. It is suggested that the ISApl1 is a key component in the mobilization of the mcrgene.ISApl1 belongs to theIS30 family and was first identified in Actinobacillus pleuropneumoniae, a Gram-negative bacterium of the Pasteurellaceae family, causing pig necrotic pleuropneumonia ${ }^{42,49,54}$.

It is also suggested that the $m c r-1$ gene had been initially mobilized by two copies of ISApl1 from an unknown progenitor. Over the course of evolution, this complex transposon has lost one or both copies of ISApl1, since it is common for IS30 family member to lose a copy of the IS element by transposition or illegitimate recombination events, in order to stabilize the mcr-1 bearing plasmid. Sometimes the $m c r-1$ gene can be mobilized by only a single copy of ISAPI1 upstream the gene in combination to an incomplete sequence of the ISApl1 downstream. ISApl1 is presents in multiple copies in the genome and these insertion sequence sites are notable for their high AT content ${ }^{49,52,55,56}$.

As already mentioned, the $m c r-1$ gene was originally described mostly among $E$. coli isolates from animal sources and this indicates that this gene is of animal origin. The genetic context of the $m c r-1$ gene reinforces the theory that the gene was originally from isolates obtained from animals. The first evidence is that the $m c r-1$ gene is very often associated to the insertion sequence ISApl1 and this insertion sequence is usually identified in bacteria of animal origin. Another indicative of the animal origin and the genetic context of the $m c r-1$ is the presence of other antibiotic resistance genes specific to veterinary medicine, such as the floR gene which confers resistance to florfenicol ${ }^{57}$.

Moreover, the Moraxella genus was identified as potential reservoirs of $m c r$-like genes that might be mobilized from their original host to become an acquired resistance mechanism in clinically significant species. The $m c r-1$ and $m c r-2$ genes present a significant identity with intrinsic chromosomal genes of the Moraxella species. The exact species of the progenitors of $m c r-1$ and $m c r-2$ genes remains to be determined, but the most closely-related variant compared to MCR-1 was identified in Moraxella porci, while the most closely-related variant of MCR-2 was identified in Moraxella osloensis ${ }^{57}$.

\section{Detection of MCR-Producers}

Broth microdilution (BMD) is considered the reference test for determining the susceptibility profile of polymyxins regardless the fact that BMD do not establish the underlying mechanism of resistance ${ }^{58}$. There are a few phenotypic tests which are supposedly able to detect MCR producers, including: the Colistin MAC Test (CMT) ${ }^{59}$; Combined Disc Test (CDT); Colistin MIC Reduction (CMR); Modified Rapid Polymxin NP Test (modified-RPNP) and alteration of Zeta potential tests ${ }^{60}$. Nevertheless, the molecular tests are considered the reference tests for the detection of the $\mathrm{mcr}$ gene; the molecular techniques using sequencing can also specify the gene variants.

Assays based on colistin MIC reduction in the presence of dipicolinic acid (DA) or ethylene diamine tetra-acetic acid (EDTA) have been evaluated to detect $m c r$-producing Enterobacterales $^{59,60}$. These tests are based on the fact that the catalytic domains of the MCR enzymes resemble that of zinc metalloproteins ${ }^{61}$. DA or EDTA are able to chelate zinc (necessary for the enzymatic activity of the PEtN transferase) and consequently can inhibit the enzymatic activity of MCR-1 and finally reduce colistin resistance in MCR-producing strains.

The CMT method is a broth microdilution method, in which colistin MIC $(0.125$ to $8.0 \mu \mathrm{g} / \mathrm{mL})$ is tested in absence and in presence of fixed concentration of the DA $(900 \mu \mathrm{g} /$ $\mathrm{mL}$ ). If the MIC increases $\geq 8$-fold, in the presence of DA, the result is interpreted as $m c r$-positive while an MIC reduction of $\leq 2$-fold is interpreted as $m c r$-negative. The test does not present satisfactory results with $K$. pneumoniae, probably due to a reduced permeability to DA and/or the presence of additional unknown mechanisms. In addition, the authors tested the effect of DA in increasing colistin susceptibility of $m c r-1$ positive isolates, in a disc-diffusion format, however no significant difference was detected as the inhibition zones between $\mathrm{mcr}$-1-positive and $\mathrm{mcr}$-negative isolates were very similar ${ }^{59}$. 
Similarly, Esposito et al. (2017) ${ }^{60}$ evaluated four EDTAbased assays to detect MCR-1-positive isolates. The CDT test compare the inhibition zones of colistin $(10 \mu \mathrm{g})$ and colistin plus EDTA $(100 \mathrm{mM})$. An incremental difference of $\geq 3 \mathrm{~mm}$ between the colistin impregnated disc and the colistin-EDTA-impregnated disc was interpreted as MCR-1positive. The CDT presented sensitivity and specificity of $96.7 \%$ and $89.6 \%$, respectively ${ }^{60}$.

The CMR test was designed according to the BMD method with or without the addition of $80 \mu \mathrm{g} / \mathrm{ml}$ EDTA, into the wells containing $0.06-32 \mu \mathrm{g} / \mathrm{ml}$ of colistin. $\mathrm{A} \geq$ 4-fold colistin MIC reduction in EDTA-containing wells was interpreted as MCR-1-positive. The sensitivity and specificity of this method was $96.7 \%$ and $83.3 \%$, respectively ${ }^{60}$.

The original RPNP test consists of detecting bacterial growth on a glucose based medium, in the presence of a defined concentration of colistin. The bacterial growth leads to acid formation in the medium which alters the $\mathrm{pH}$ and this can be detected visually by a $\mathrm{pH}$ indicator color change ${ }^{62}$. If the well containing colistin, change its colour from orange to yellow, it is considered positive (colistin resistance). The modified RPNP is based on the incorporation of two extra wells, one containing $80 \mu \mathrm{g} / \mathrm{mL}$ EDTA (without colistin) and the other one containing $80 \mu \mathrm{g} / \mathrm{ml}$ EDTA plus $5 \mu \mathrm{g} / \mathrm{ml}$ colistin. In this case, the MCR-1 positive result is observed when the colistin containing solution supplemented with EDTA remained orange (i.e., absence of glucose metabolization due to EDTA inhibition). The sensitivity and specificity of this test are higher (96.7\% and $100.0 \%$, respectively) compared to CDT and CMR tests ${ }^{60}$.

Another method for phenotypic detection of $\mathrm{mcr}$ producers is the alteration of Zeta potential in the absence and presence of $80 \mu \mathrm{g} / \mathrm{ml}$ EDTA, which increase the anionic charges on the surface membrane of MCR-1-positive isolates. However, according to the authors, this method can only be used in well-resourced microbiology laboratories ${ }^{60}$.

Polymerase Chain Reaction (PCR) and Whole Genome Sequence (WGS) are considered the reference tests to identify the $m c r$ gene from cultured bacteria as well as in clinical, fecal, environmental and food samples ${ }^{58,63}$. While PCR can only detect known mor genes, due to specific primers and probes, the WGS can identify all known or unknown colistin resistance mechanisms within 2 days ${ }^{58}$. Further molecular tests are the commercial microarray that can simultaneously detect both $\beta$-lactamases and $m c r-1 /-2$ genes, even though those tests are inaccessible to under-resourced laboratories ${ }^{64}$.

\section{Conclusion}

Considering the importance of polymyxins in human and veterinary medicine, there is an urgent need to limit the propagation of $m c r-1$ harboring plasmid. Given the constant exchange of resistance genes in all microbiomes (animals, environment and human population), surveillance programs to monitor the diversity of reservoirs, plasmids and to evaluate the real dimension of resistance mediated by the $m c r$ gene are necessary.

\section{Funding Information}

This work was supported by Fundo de Incentivo a Pesquisa e Eventos do Hospital de Clínicas de Porto Alegre (FIPE/HCPA) (project no.16-0559) and by a grant from the Conselho Nacional de Desenvolvimento Científico e Tecnológico-Brazil (CNPq).

\section{References}

1. Liu YY, Wang Y, Walsh TR, et al. Emergence of plasmid-mediated colistin resistance mechanism MCR-1 in animals and human beings in China: a microbiological and molecular biological study. Lancet Infect Dis. 2016; 16: 161-8

2. Irrgang A, Roschanski N, Tenhagen BA, et al. Prevalence of mcr-1 in E. coli from livestock and food in Germany, 2010-2015. PlosOne. 2016; 11 (7): e0159863.

3. Nordmann P, Jayol A, Poirel L, et al. A universal culture medium for screening polymyxin-resistant Gram-negative isolates. J Clin Microbiol. 2016; 54 (5): 1395-9.

4. Sherry N, Howden B. Emerging Gram-negative resistance to last-line antimicrobial agents fosfomycin, colistin and ceftazidime-avibactam - epidemiology, laboratory detection and treatment implications. Expert Rev Anti Infect Ther. 2018; 16 (4): 289-306.

5. Poirel L, Jayol A, Nordmann P, et al. Polymyxins: antibacterial activity, susceptibility testing, and resistance mechanisms encoded by plasmids or chromosomes. Clin Microbiol Rev. 2017; 30 (2): 557-96.

6. Sader HS, Rhomberg PR, Farrell DJ, et al. Differences in potency and categorical agreement between colistin and polymyxin $B$ when testing 15,377 clinical strains collected worldwide. Diagn Microbiol Infect Dis. 2015; 83 (4): 379-81.

7. Srinivas P, Rivard K. Polymyxin Resistance in Gram-negative Pathogens. Curr Infect Dis Rep. 2017; 19: 38.

8. Benedict RG, Langlykke AF. Antibiotic activity of Bacillus polymyxa. J Bacteriol. 1947; 54: 24

9. Skov RL, Monnet DL. Plasmid-mediated colistin resistance (mcr-1 gene): three months later, the story unfolds. Euro Surveill. 2016; 21 (9): 30155.

10. Walsh TR, Wu Y. China bans colistin as a feed additive for animals. Lancet Infect Dis. 2016; 16 (10): 1102-3.

11. Zavascki AP, Goldani LZ, Li J, et al. Polymyxin B for the treatment of multidrug-resistant pathogens: a critical review. J Antimicrob Chemother. 2007; 60 (6): 1206-15.

12. Ye H, Li Y, Li Z, et al. Diversified mcr-1-harbouring plasmid reservoirs confer resistance to colistin in human gut microbiota. mBio. 2016; 7 (2): e00177-16.

13. Prim N, Rivera A, Rodríguez-Navarro J, et al. Detection of mcr- 1 colistin resistance gene in polyclonal Escherichia coli isolates in Barcelona, Spain, 2012 to 2015. Euro Surveill. 2016; 21 (3): 30183.

14. Shen Z, Wang Y, Shen Y, et al. Early emergence of mcr-1 in Escherichia coli from food-producing animals. Lancet Infect Dis. 2016; 16 (3): 293.

15. Fernandes MR, Moura Q Sartori L, et al. Silent dissemination of colistin-resistant Escherichia coli in South America could contribute to the global spread of the mcr-1 gene. Euro Surveill. 2016; 21 (17): 30214 . 
16. Lentz SA, de Lima-Morales D, Cuppertino VM, et al. Letter to editor: Escherichia coli harbouring mcr-1 gene isolated from poultry not exposed to polymyxins in Brazil. Euro Surveill. 2016; 21 (26): 1-2.

17. Pham Thanh D, Thanh Tuyen H, Nguyen Thi Nguyen T, et al. Inducible colistin resistance via a disrupted plasmid-borne mcr-1 gene in a 2008 Vietnamese Shigella sonnei isolate. J Antimicrob Chemother. 2016; 71(8): 2314-7.

18. Terveer EM. Prevalence of colistin resistance gene (mcr1) containing Enterobacteriaceae in feces of patients attending a tertiary care hospital and detection of a mcr-1 containing colistin susceptible E. coli. PLoS ONE. 2017; 12 (6): e0178598.

19. Liassine N, Assouvie L, Descombes MC, et al. Very low prevalence of MCR-1/MCR-2 plasmid-mediated colistin resistance in urinary tract Enterobacteriaceae in Switzerland. Int J infect Dis. 2016; 51: 4-5.

20. Di Pilato V, Arena F, Tascini C, et al. mcr-1.2, a new mcr variant carried on a transferable plasmid from a colistin-resistant KPC carbapenemase-producing Klebsiella pneumoniae strain of sequence type 512. Antimicrob Agents Chemother. 2016; 60: 5612-5.

21. Lu X. mcr-1.3: a new mcr variant carried by an IncP plasmid in a colistin-resistant Salmonella enterica serovar Typhimurium isolated from a healthy individual. Antimicrob Agents Chemother. 2017. In press.

22. Yang YQ, Li YX, Song T, et al. Colistin resistance gene mcr-1 and its variant in Escherichia coli isolates from chickens in China. Antimicrob Agents Chemother. 2017; 61 (5): e01204-16.

23. Tijet N, Faccone D, Rapoport M, et al. Molecular characteristics of mcr1-carrying plasmids and new mcr-1 variant recovered from polyclonal clinical Escherichia coli from Argentina and Canada. PlosOne. 2017; 12 (7): $\mathrm{e} 0180347$.

24. Liu H, Zhu B, Liang B, et al. A Novel mcr-1 variant carried by an Incl2-Type plasmid identified from a multidrug resistant enterotoxigenic Escherichia coli. Front Microbiol. 2018; 9: 815.

25. AbuOun M, Stubberfield EJ, Duggett NA, et al. mcr-1 and mcr-2 variant genes identified in Moraxella species isolated from pigs in Great Britain from 2014 to 2015. J Antimicrob Chemother. 2017; 72 (10): 2745-9.

26. Alba P, Leekitcharoenphon P, Franco A, et al. Molecular epidemiology of mcr-encoded colistin resistance in Enterobacteriaceae from foodproducing animals in Italy revealed through the eu harmonized antimicrobial resistance monitoring. Front Microbiol. 2018; 9: 1217.

27. Xavier BB, Lammens C, Ruhal R, et al. Identification of a novel plasmidmediated colistin resistance gene, mcr-2, in Escherichia coli, Belgium, June 2016. Euro Surveill. 2016; 21 (27): 30280.

28. Yin W, Li H, Shen Y, et al. Novel plasmid-mediated colistin resistance gene mcr-3 in Escherichia coli. MBio. 2017; 8 (3): e00543-17.

29. Carattoli A, Villa L, Feudi C, et al. Novel plasmid-mediated colistin resistance mcr-4 gene in Salmonella and Escherichia coli, Italy 2013, Spain and Belgium, 2015 to 2016. Euro Surveill. 2017; 22 (31): 30589

30. Borowiak M, Fischer J, Hammerl JA, et al. Identification of a novel transposon-associated phosphoethanolamine transferase gene, mcr5 , conferring colistin resistance in d-tartrate fermenting Salmonella enterica subsp. enterica serovar Paratyphi B. J Antimicrob Chemother. 2017; 72: 3317-24.

31. Yang YQ Li YX, Lei CW, et al. Novel plasmid-mediated colistin resistance gene mcr-7.1 in Klebsiella pneumoniae. J Antimicrob Chemother. 2018; 73 (7): 1791-5.

32. Wang X, Wang Y, Zhou Y, et al. Emergence of a novel mobile colistin resistance gene, mcr-8, in NDM-producing Klebsiella pneumoniae. Emerg Microbes Infect. 2018; 7 (1): 122.

33. Hasman H, Hammerum AM, Hansen F, et al. Detection of mcr1 encoding plasmid-mediated colistin-resistant Escherichia coli isolates from human bloodstream infection and imported chicken meat, Denmark. Euro Surveill. 2016; 20 (49): 30085.

34. Rapoport M, Faccone D, Pasteran F, et al. mcr-1-mediated colistin resistance in human infections caused by Escherichia coli: First description in Latin America. Antimicrob Agents Chemother. 2016; 60 (7): 4412-2

35. Li Y, Sun QL, Shen Y, et al. Rapid increase in prevalence of carbapenemresistant Enterobacteriaceae (CRE) and emergence of colistin resistance gene mcr-1 in CRE in a hospital in Henan, China. J Clin Microbiol. 2018; 56(4): e01932-17.

36. Wang R, Liu Y, Zhang Q, et al. The prevalence of colistin resistance in Escherichia coli and Klebsiella pneumoniae isolated from food animals in China: coexistence of mcr-1 and bla vpm $_{\text {with low fitness }}$ cost. Int J Antimicrob Agents. 2018; 51(5): 739-44.

37. Bulman ZP, Chen L, Walsh TJ, et al. Polymyxin combinations combat Escherichia coli harboring mcr- 1 and bla $_{\mathrm{NDM}-5}$ : preparation for a postantibiotic era. MBio. 2017; 8 (4): e00540-17.

38. Liu YY, Chandler CE, Leung LM, et al. Structural modification of lipopolysaccharide conferred by $\mathrm{mcr}-1$ in Gram-negative ESKAPEPathogens. Antimicrob Agents Chemother. 2017; 61 (6): e00580-17.

39. Du H, Chen L, Tang YW, et al. Emergence of the mcr-1 colistin resistance gene in carbapenem-resistant Enterobacteriaceae. Lancet Infect Dis. 2016; 16 (3): 287-8

40. Mulvey MR. Dissemination of the mcr-1 colistin resistance gene. Lancet Infect Dis. 2016; 16 (3): 289-90.

41. Poirel L, Kieffer N, Liassine N, et al. Plasmid-mediated carbapenem and colistin resistance in a clinical isolate of Escherichia coli. Lancet Infect Dis. 2016; 16 (3): 281.

42. Falgenhauer L, Waezsada SE, Yao Y, et al. Colistin resistance gene mcr1 in extended spectrum $\beta$-lactamase producing and carbapenemase producing Gram-negative bacteria in Germany. Lancet Infect Dis. 2016; 16 (3): 280.

43. Conceição-Neto OC, Aires CAM, Pereira NF, et al. Detection of the plasmid-mediated mcr-1 gene in clinical KPC-2-producing Escherichia coli isolates in Brazil. Int J Antimicrob Agents. 2017; 50 (2): 282-4.

44. Dalmolin TV, Castro L, Mayer FQ, et al. Co-occurrence of mcr-1 and $\mathrm{bla}_{\mathrm{KPC}-2}$ in a clinical isolate of Escherichia coli in Brazil. J Antimicrob Chemother. 2017; 72 (8): 2404-6

45. Tacão M, Tavares RDS, Teixeira P, et al. mcr-1 and bla $\mathrm{KPC}-3_{3}$ in Escherichia coli Sequence Type 744 after meropenem and colistin therapy, Portugal. Emerg Infect Dis. 2017; 23(8): 1419-21.

46. Zhao D, Zhou Z, Hua X, et al. Coexistence of mcr-1, bla $_{\mathrm{KPC}-2}$ and two copies of fosA3 in a clinical Escherichia coli strain isolated from urine. Infect Genet Evol. 2018; 60:77-9.

47. Aires CAM, da Conceição-Neto OC, Tavares E Oliveira TR, et al. Emergence of the plasmid-mediated mcr-1 gene in clinical KPC2-producing Klebsiella pneumoniae Sequence Type 392 in Brazil. Antimicrob Agents Chemother. 2017; 61 (7): e00317-17.

48. Dalmolin TV, Martins AF, Zavascki AP, et al. Acquisition of the mcr-1 gene by a high-risk clone of KPC-2-producing Klebsiella pneumoniae ST437/CC258, Brazil. Diagn Microbiol Infect Dis. 2018; 90 (2): 132-3.

49. Poirel L, Kieffer N, Nordmann P. In vitro study of ISApl1-mediated mobilization of the colistin resistance gene mcr-1. Antimicrob Agents Chemother. 2017; 61 (7): e00127-17.

50. Campos J. MCR-1 in multidrug-resistant and copper-tolerant clinically relevant Salmonella 1, 4, [5], 12: I : - and S. Rissen clones in Portugal, 2011 to 2015. Euro Surveill. 2016; 21 (26): 30270.

51. Tse H, Yuen KY. Dissemination of the mcr-1 colistin resistance gene. Lancet Infect Dis. 2016; 16 (2): 145-6. 
52. Zurfluh K, Nüesch-Inderbinen M, Klumpp J, et al. Key features of mcr1 bearing plasmids from Escherichia coli isolated from humans and food. Antimicrob Resist Infect Control. 2017; 6: 91.

53. Liu L, Feng Y, Zhang X, et al. New variant of mcr-3 in an extensively drug-resistant Escherichia coli clinical isolate carrying mcr-1 and bla $_{\mathrm{NDM}-5^{\circ}}$. Antimicrob Agents Chemother. 2017; 61 (12): e01757-17.

54. Petrillo M, Angers-Loustau A, Kreysa J. Possible genetic events producing colistin resistance gene mcr-1. Lancet Infect Dis. 2016; 16 (3): 280.

55. Snesrud E, Ong AC, Corey B, et al. Analysis of serial isolates of mcr1-positive Escherichia coli reveals a highly active ISApl1transposon. Antimicrob Agents Chemother. 2017; 61 (5): e00056-17.

56. Snesrud E, He S, Chandler M, et al. A model for transposition of the colistin resistance gene mcr-1 by ISApl1. Antimicrob Agents Chemother. 2016; 60 (11): 6973-6.

57. Kieffer N, Nordmann P, Poirel L. Moraxella species as potential sources of MCR-like polymyxin resistance determinants. Antimicrob Agents Chemother. 2017; 61 (6): e00129-17.

58. Sekyere JA, Asante J. Emerging mechanisms of antimicrobial resistance in bacteria and fungi: advances in the era of genomics. Future Microbiol. 2018; 13: 241-62.
59. Coppi M, Cannatelli A, Antonelli A, et al. A simple phenotypic method for screening of MCR-1-mediated colistin resistance. Clin Microbiol Infect. 2018; 24 (2): 201.

60. Esposito F, Fernandes MR, Lopes R, et al. Detection of colistinresistant MCR-1-positive Escherichia coli by use of assays based on inhibition by EDTA and Zeta Potential. J Clin Microbiol. 2017; 55 (12): 3454-65.

61. Hinchliffe P, Yang QE, Portal E, et al. Insights into the mechanistic basis of plasmid-mediated colistin resistance from crystal structures of the catalytic domain of MCR-1. Sci Rep. 2017; 7: 39392.

62. Nordmann P, Jayol A, Poirel L. Rapid Detection of polymyxin resistance in Enterobacteriaceae. Emerg Infect Dis. 2016; 22 (6): 1038-43.

63. Rebelo AR, Bortolaia V, Kjeldgaard JS, et al. Multiplex PCR for detection of plasmid-mediated colistin resistance determinants, mcr1, mcr-2, mcr-3, mcr-4 and mcr-5 for surveillance purposes. Euro Surveill. 2018; 23 (6): 180215-1.

64. Bernasconi OJ, Principe L, Tinguely R, et al. Evaluation of a new commercial microarray platform for the simultaneous detection of beta-lactamase and mcr-1 and mcr-2 genes in Enterobacteriaceae. J Clin Microbiol. 2018; 55: 3138-41. 